\title{
Long-term results of the surgical treatment of Peyronie's disease with Egydio's technique: a European multicentre study
}

\author{
Salvatore Sansalone ${ }^{1}$, Giulio Garaffa ${ }^{2}$, Rados Djinovic ${ }^{3}$, Stefano Pecoraro ${ }^{4}$, Mauro Silvani ${ }^{5}$, Guido Barbagli ${ }^{6}$, \\ Alessandro Zucchi $^{7}$, Giuseppe Vespasiani ${ }^{1}$ and Carla Loreto ${ }^{8}$
}

The long-term outcomes of 157 patients affected by Peyronie's disease (PD) who underwent penile straightening with Egydio's technique between January 2004 and December 2008 are reported. Only patients with PD who were stable for at least 6-12 months prior to surgery were enrolled in this study. Preoperative assessment included a dynamic echo colour Doppler ultrasound scan to evaluate the degree of penile deformity and the peak systolic velocity in the cavernosal arteries and an assessment of erectile function with the administration of the International Index of Erectile Function 5 (IIEF-5) questionnaire. Stretched penile length was recorded pre- and postoperatively. Surgical complications, cosmesis and sexual function, patient satisfaction and postoperative erectile function were assessed postoperatively at 3 months, 1 year and 2 years, respectively. After a median follow-up period of 20 months (range: 12-24 months), we found that mild residual curvature (12\%) and glans hypoesthesia (3\%) were the only causes of partial dissatisfaction. No rejection of the graft was observed. All patients recovered their ability to penetrate with no difficulty. In addition, an intraoperative average increase of $2.5 \mathrm{~cm}$ (range: $1.7-4.1 \mathrm{~cm}$ ) in stretched penile length was recorded, with all patients engaging in penetrative sexual intercourse. In conclusion, this procedure represents a safe and reproducible technique for the correction of penile curvature resulting from PD and yields excellent cosmetic and functional results.

Asian Journal of Andrology (2011) 13, 842-845; doi:10.1038/aja.2011.42; published online 11 July 2011

Keywords: Egydio's technique; erectile dysfunction; graft; Peyronie's disease

\section{INTRODUCTION}

Peyronie's disease $(\mathrm{PD})$ is currently considered a wound-healing disorder that results in a fibrous inelastic scar of the tunica albuginea. The condition, which is believed to occur in genetically susceptible individuals following penile trauma, is characterized by the formation of a palpable scar that does not stretch in the erect state along with the remaining tunica albuginea. This scar can cause a variety of deformities, including curvature, shortening, narrowing and a hinge effect. In the early phase, there is often an inflammatory component that causes pain.

$\mathrm{PD}$ is also frequently associated with erectile dysfunction (ED) and a variety of other comorbid disorders, including diabetes, hypertension, dyslipidemia and low testosterone. The quality of life of both the patient and his partner may be considerably affected, with an increased risk of depression, low self-esteem and relationship difficulties. ${ }^{1-3}$

$\mathrm{PD}$ is a progressive disorder, with up to $48 \%$ of men experiencing disease progression if left untreated. In most cases, PD may be divided into an acute inflammatory phase and a chronic phase. During the acute inflammatory phase, there may be penile pain and curvature progression, although the pain typically resolves spontaneously within 6-18 months from onset in most patients. ${ }^{4}$

Surgery is indicated only in the chronic phase if the curvature impedes adequate sexual penetration or if there is associated ED that fails to respond to medical treatment. Surgery should be offered only once the disease has stabilized. ${ }^{5,6}$

Among the possible surgical options, plaque incision or partial plaque excision and grafting is indicated in patients with adequate rigidity, with or without drug assistance, when there is simple curvature of more than $60-70^{\circ}$, when there is complex or hourglass deformity, and when the presumed loss of length caused by the plication is more than $20 \%$ of the total erect length. ${ }^{1}$

\section{MATERIALS AND METHODS}

From January 2004 to December 2008, 157 patients with PD underwent plaque incision and grafting according to the geometrical principles described by Egydio et al. ${ }^{7-9}$ All patients had had stable disease for at least 6-12 months at the time of surgery.

${ }^{1}$ Department of Urology, School of Medicine Tor Vergata University of Rome, Rome 00133, Italy; ${ }^{2}$ St Peter's Andrology, University College London Hospitals, London W1G 6BJ, UK; ${ }^{3}$ Department of Urology, School of Medicine, Serbian Academy of Science and Arts, University of Belgrade, Belgrade 11000, Serbia; ${ }^{4}$ Department of Nephro-Urology, Malzoni Medical Center Avellino, Avellino 83100, Italy; ${ }^{5}$ Department of Urology, General Hospital, Biella 13900, Italy; ${ }^{6}$ Center for Reconstructive Urethral Surgery, Arezzo 52100, Italy; ${ }^{7}$ Department of Urology and Andrology, University of Perugia, Perugia 06123, Italy and ${ }^{8}$ Department of Bio-Medical Sciences, Anatomy Section, University of Catania, Catania 95100, Italy 
Preoperative assessment of the quality of the erection included a dynamic echo colour Doppler ultrasound scan after the administration of 10-20 $\mu \mathrm{g}$ of prostaglandin E1 and the administration of the International Index of Erectile Function 5 (IIEF-5) questionnaire. All patients with an IIEF score of less than 15 and a peak systolic velocity (PSV) of less than $35 \mathrm{~cm} \mathrm{~s}^{-1}$ were counselled against undergoing the operation and were offered penile prosthesis implantation.

Surgery was carried out at three Italian centres by SS, SP and MS on 56 patients and in Belgrade by RD on the remaining patients. The median age at the time of surgery was $55 \pm 11$ years (range: $29-70$ years), the median preoperative IIEF-5 score was 16 (range: 15-21) and the degree of curvature, assessed intraoperatively with an artificial erection, is reported in Table $\mathbf{1}$.

In all patients, penetration was extremely difficult or impossible due to the degree of the curvature.

As previously described by Egydio et al., ${ }^{7-9}$ the penis was degloved using a circumferential subcoronal incision and an artificial erection was induced to assess the degree of deformity and the point of maximal curvature (Figure 1a). After an accurate dissection of the Buck's fascia, an incomplete circumferential double $\mathrm{Y}$ incision of the tunica albuginea was carried out at the point of maximal curvature.

For dorsal, dorsolateral and lateral curvatures, this incision in the tunica albuginea was made as far as the lateral borders of the urethra, which was not mobilized; for ventral or ventrolateral curvatures, the urethra was dissected off the corpora cavernosa, and an incomplete circumferential incision was made almost to the deep dorsal vein (Figure 1b).

A 3- to 5-mm dissection was performed with a scalpel between the tunica and spongy tissue of the cavernous body, and the septum was incised in all patients to relax the scarred tissue and to transform the linear tunical incision into a rectangular defect, thus allowing a lengthening of the short (concave) side of the shaft. A small fragment of plaque has been excised in all cases and sent for histopathological confirmation of Peyronie's disease. With the penis under traction, the size of the defect in the tunica was measured, and the defect was covered with a bovine pericardium collagen matrix graft (Veritas ${ }_{\circledR}$ Collagen Matrix; Synovis Life Technologies, Inc., St Paul, MN, USA) that was sutured to the tunica albuginea using a continuous 4-0 Poliglecaprone® suture.

The complete straightening of the penis was confirmed intraoperatively with an artificial erection in all patients, and the stretched penile length was assessed preoperatively and at the completion of the surgical procedure (Figure 1c). In all patients, the curvature was completely corrected with Egydio's procedure, and therefore, no contralateral plication was necessary.

Patients were discharged on postoperative day 1 and were recommended to refrain from any form of sexual intercourse for 6 weeks.

An artificial erection was induced with an intracavernosal injection of prostaglandin E1 3 months postoperatively, and patient and partner satisfaction and erectile function were assessed postoperatively at 3 months, 1 year and 2 years, respectively. The IIEF- 5 questionnaire was administered postoperatively in 89 patients to assess erectile function.

Table 1 Type and degree of curvature of the intraoperative artificial erection

\begin{tabular}{lccccc}
\hline Location & Dorsal & Lateral & Dorsolateral & Ventral & Total patients \\
\hline Belgrade $(n)$ & 73 & 10 & 10 & 8 & 101 \\
Italy $(n)$ & 39 & 9 & 7 & 1 & 56 \\
Total $(n)$ & 112 & 19 & 17 & 9 & 157 \\
\hline
\end{tabular}
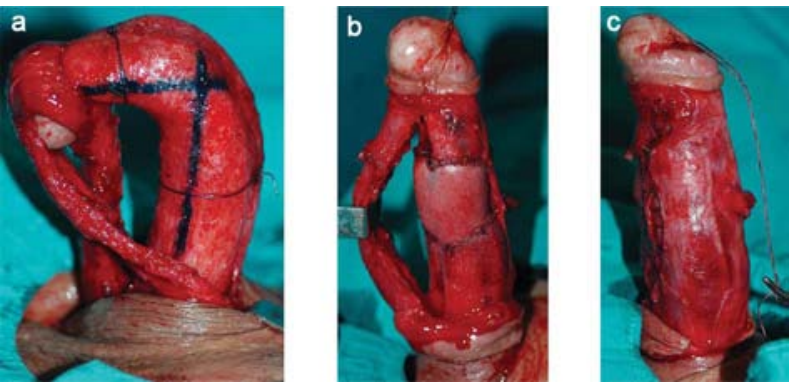

Figure 1 Step by step description of the technique (a) The penis is degloved, and the degree of curvature is assessed with an artificial erection after accurate dissection of the urethra and neurovascular bundle. (b) The ventral curvature is corrected with a relaxing double $Y$ incision that is carried out almost to the dorsal vein complex, and the defect is grafted with a bovine pericardium collagen matrix graft. (c) The complete correction of the curvature is documented with an artificial erection.

\section{RESULTS}

After a median follow-up period of 20 months (range: 12-24 months), no rejection of the graft was observed. Minor residual curvature (less than $15^{\circ}$ ) that did not interfere with penetrative intercourse was detected 3 months postoperatively after induction of an artificial erection in 19 patients (12\%). In all patients, the intraoperative artificial erection had shown a complete correction of the penile curvature. Mild glans hypoesthesia was reported by five patients (3\%) and, together with the residual curvature, represented the only cause of partial dissatisfaction.

Overall, the median gain in penile length was $2.5 \mathrm{~cm}$ (range: 1.5$3.5 \mathrm{~cm}$ ), and the median postoperative IIEF-5 score showed a significant improvement, increasing from the preoperative 16.0 (range: 15.0-21.0) to 18.5 (range: 17.0-23.0), 20.5 (range: 19.0-24.0) and 22.5 (range: 20.0-25.0), respectively, at 3-, 12- and 24-month follow-up.

One year after surgery, all 157 patients were able to have normal sexual intercourse, and only $29 \%$ required phosphodiesterase type 5 inhibitors to achieve adequate rigidity; the percentage increased to $32 \%$ after 2 years of operation.

Overall, $97 \%$ of partners were fully satisfied with the cosmetic and functional outcomes of the surgery.

\section{DISCUSSION}

The quest for the ideal surgical treatment for PD has constituted a challenge. The variety of techniques proposed shows that no consensus exists as to a single procedure that meets the needs of most patients and reflects the fact that the presentation of $\mathrm{PD}$ varies widely among patients in terms of the degree of deformity, penile shortening and residual erectile function.

Surgery should be offered only once the disease has stabilized and is indicated when the curvature impedes adequate penetrative intercourse or if there is associated ED that fails to respond to medical treatment.

Surgical grafting techniques include plaque incision or partial excision and are indicated in patients with adequate preoperative erectile function and when there is a simple curvature of more than $60-70^{\circ}$, when there is complex or hourglass deformity, and when the presumed loss of length caused by the plication will be more than $20 \%$ of the total erect length.

Patients with poor-quality erections are instead better treated with penile prosthesis implantation. ${ }^{1}$ Penile prosthesis implantation alone may result in satisfactory straightening of the penis in patients with 
mild deformity, but when residual curvature is greater than $20^{\circ}$, additional manoeuvres such as manual modelling, plication and tunical incision with or without grafting are required to achieve complete straightening. ${ }^{1-10}$ Manual modelling is initially attempted for $90 \mathrm{~s}$ and can be repeated a second time; if residual curvature persists, complete straightening can be achieved with plication of the convex side or performing a relaxing tunical incision on the concave aspect of the shaft. Grafting is indicated if the incisions create a defect greater than $2 \mathrm{~cm}^{1}$

Historically, total plaque excision was designed to "remove the diseased tunica", but total plaque excision results in an unacceptably high rate of postoperative $\mathrm{ED}$, probably as a result of a compromised veno-occlusive mechanism because of the changes in the relationship between the cavernosal tissue and the overlying tunica or graft. ${ }^{11}$ Therefore, it is recommended to minimize the size of the excision so that a smaller graft can be used. ${ }^{12}$

To date, there has been no randomized controlled trial in humans comparing different types of graft materials and surgical outcomes; however, animal studies on the use of different graft materials for tunical substitution have shown satisfactory outcomes in cadaveric pericardial, dermal and vein grafts compared with synthetic grafts.

Although an ideal graft material that would take reliably and would not contract, would be resistant to infection, and would preserve erectile capacity has not yet been identified, currently it appears that the nature of the graft is not likely to be the determining factor with respect to postoperative $\mathrm{ED} .^{13,14}$

It is more likely that ED is a result of patient selection with respect to preoperative erectile status and operative technique. In particular, a higher risk of ED was found in those who reported preoperative diminished rigidity, were over the age of 60 years, required larger grafts or underwent grafting for ventral curvature. ${ }^{15}$

Various authors have reported their experience with plaque incision and grafting; their results in terms of postoperative residual curvature and new-onset ED are reported in Table 2. ${ }^{12,16-26}$ Overall, 22\%-100\% of patients were adequately straight, with the incidence of postoperative ED ranging from $5 \%$ to $67 \%$.

The addition of phosphodiesterase type 5 inhibitors or other pharmacological erectile agents postoperatively is common, while the risk of postoperative ED appears to be higher in men who receive pharmacologic erectile agents prior to surgery. ${ }^{25}$

In the present study, the postoperative addition of phosphodiesterase type 5 inhibitors was necessary in $32 \%$ of patients. The postoperative increase in the score of the IIEF- 5 was in domains 3,4 and
5 and reflected the increased confidence of the patients who had regained a straight penis and were able to engage in penetrative sexual intercourse. Only 89 patients completed the IIEF-5 questionnaire postoperatively, which is a limitation of this study. However, all patients were able to engage successfully in penetrative sexual intercourse 2 years postoperatively.

The present study confirms that plaque incision and grafting using Egydio's technique yields excellent functional results; all patients reported that they were able to engage in penetrative sexual intercourse, and the penis was completely straight in $88 \%$ of cases.

Moreover, this series shows that severe postoperative ED can be prevented with an adequate preoperative selection of patients and if the correction of the curvature is alleviated with a single tunical incision without excision of the plaque, as the size of the graft has a significant impact on postoperative erectile function. ${ }^{15}$

Furthermore, this series confirms that a double $\mathrm{Y}$ relaxing incision without plaque excision described, as by Egydio et al., ${ }^{9}$ allows the correction of all types of curvature, whether or not the curvature is associated with an hourglass deformity. Plaque excision techniques should be avoided not only because they are associated with a high incidence of ED but also because they frequently demand complementary relaxing incisions to correct the curvature despite of the removal of the plaque. This confirms that the damage to the tunica is diffuse and not simply restricted to the plaque.

Various types of relaxing incisions have been described for each type of curvature, such as the ' $\mathrm{H}$ '-shaped incision described by various authors. ${ }^{1,20,21,27}$ However, while the circumferential double $Y$ incision guarantees complete correction of virtually any type of deformity because it breaks all of the lines of force, the ' $\mathrm{H}$ '-shaped incision induces only partial relaxation, and, therefore, multiple incisions and/or plications are necessary to achieve complete correction in patients with more complex curvature.

\section{CONCLUSION}

The present study confirms the long-term reliability of Egydio's technique, which is simple and very reproducible. However, adequate preoperative selection of patients is necessary, and patients must be warned that long-term worsening of the quality of the erection may occur in up to one-third of cases.

\section{AUTHOR CONTRIBUTIONS}

GB and GG have designed the study. SS, SP, MS and RD have carried out the surgical procedures, while $\mathrm{AZ}, \mathrm{GV}$ and $\mathrm{CL}$ have

Table 2 Outcome of plaque incision and grafting

\begin{tabular}{|c|c|c|c|c|c|}
\hline Author, Year & No. of patients & Type of graft & Straight (\%) & $E D(\%)$ & Follow-up (month) \\
\hline Gelbart, $1996^{12}$ & 69 & Temporalis fascia & 74 & 14 & Not reported \\
\hline Lue et al., $1998^{16}$ & 112 & Venous grafting & 96 & 12 & 18 \\
\hline Egydio et al., $2002^{9}$ & 33 & Bovine pericardium & 87 & - & 19 \\
\hline Hatzichristou et al., $2002^{17}$ & 17 & Albuginea free grafting & 100 & 0 & 39 \\
\hline Levine and Estrada, $2003^{18}$ & 40 & Human pericardium & 98 & 30 & 22 \\
\hline Montorsi et al., $2005^{19}$ & 50 & Venous graft & 88 & 22 & 60 \\
\hline Kalsi et al., $2005^{20}$ & 113 & Venous graft & 86 & 15 & 12 \\
\hline Ralph et al., $2006^{21}$ & 14 & Cadaveric fascia lata & 78 & 7 & 31 \\
\hline Knoll, $2007^{22}$ & 162 & Small intestine submucosa & 91 & 21 & 38 \\
\hline Breyer et al., $2007^{23}$ & 19 & Small intestine submucosa & 63 & 53 & 15 \\
\hline Hsu et al., $2007^{24}$ & 48 & Venous grafting & 90 & 5 & - \\
\hline Taylor and Levine, $2008^{25}$ & 101 & Pericardial graft & 92 & 35 & 58 \\
\hline Cheung et al., $2010^{26}$ & 86 & a & 22 & 67 & 98 \\
\hline
\end{tabular}

${ }^{\text {a }}$ Dermal graft ( $\left.n=20\right)$; cadaveric fascia lata ( $\left.n=33\right)$; small intestine submucosa $(n=33)$. 
collected the data. CL has carried out all the anatomical and histopathological examination of the surgical specimens. GG has carried out all the statistical analysis. All authors have read and approved the final manuscript.

\section{COMPETING FINANCIAL INTERESTS}

The authors declare that they have no competing financial interests.

1 Ralph DJ, Gonzalez-Cadavid N, Mirone V, Perovic S, Sohn M et al. The management of Peyronie's disease: evidence-based 2010 guidelines. J Sex Med 2010; 7: 2359-74.

2 Kendrici M, Trost L, Sikka SC, Hellstrom GJ. Diabetes mellitus is associated with severe Peyronie's disease. BJU Int 2007; 99: 383-6.

3 Kadioglu A, Tefekli A, Erol B, Oktar T, Tunc M et al. A retrospective review of 307 men with Peyronie's disease. J Urol 2002; 68: 1075-9.

4 Mulhall JP, Schiff J, Guhring P. An analysis of the natural history of Peyronie's disease. J Urol 2006; 175: 2115-8.

5 Bella AJ, Perelman MA, Brant WO, Lue TF. Peyronie's disease (CME). J Sex Med 2007; 4: 1527-38.

6 Kendrici M, Hellstrom WJ. Critical analysis of surgery for Peyronie's disease. Curr Opin Urol 2004; 6: 382-8.

7 Egydio PH, Lucon AM, Arap S. A single relaxing incision to correct different types of penile curvature: surgical technique based on geometrical principles. BJU Int 2004; 94: 1147-57.

8 Egydio $\mathrm{PH}$. Surgical straightening with tunical incision and grafting technique-single relaxing incision based on geometrical principles. In: Levine LA, editor. Peyronie's Disease Textbook: A Guide to Clinical Management. Totowa: Hymana Press; 2006. pp227-39.

9 Egydio PH, Lucon AM, Arap S. Treatment of Peyronie's disease by incomplete circumferential incision of the tunica albuginea and plaque with bovine pericardium graft. Urology 2002; 59: 570-4.

10 Wilson SK, Delk 2nd Jr. A new treatment for Peyronie's disease: modeling the penis over an inflatable penile prosthesis. J Urol 1994; 152: 1121-3.
11 Dakin BL, Carter MF. Venogenic impotence following dermal graft repair for Peyronie's disease. J Urol 1991; 146: 849-51.

12 Gelbard MK. Relaxing incisions in the correction of penile deformity due to Peyronie's disease. J Urol 1995; 154: 1457-60.

13 Kovac JR, Brock GB. Surgical outcomes and patient satisfaction after dermal, pericardial and small intestinal submucosal grafting for Peyronie's disease. J Sex Med 2007; 4: 1500-8.

14 Leungwattanakij S, Bivalaqua TJ, Yang DY, Hyun JS, Hellstrom WJ. Comparison of cadaveric pericardial, dermal, vein, vein, and synthetic grafts for tunica albuginea substitution using a rat model. BJU Int 2003; 92: 119-24.

15 Mulhall JP, Anderson M, Parker M. A surgical algorithm for men with combined Peyronie's disease and erectile dysfunction: functional and satisfaction outcomes. J Sex Med 2005; 2: 132-8

16 Lue TF, El Sakka Al. Venous patch graft for Peyronie's disease. Part I: Technique. J Urol 1998; 160: 2047-9.

17 Hatzichristou DG, Hatzimouratidis K, Apostolidis A, Tzortzis V, Bekos A et al. Corporoplasty using free albuginea grafts of penile curvature: surgical technique and long term results. J Urol 2002; 167: 1367-70.

18 Levine LA, Estrada CR. Human cadaveric pericardial graft for the surgical correction of Peyronie's disease. J Urol 2003; 170: 2359-62.

19 Montorsi F, Salonia A, Briganti A, Deho' F, Zanni G et al. Five year follow up of plaque incision and vein grafting for Peyronie's disease. J Urol 2004; 171: 331.

20 Kalsi J, Minhas S, Christopher N, Ralph D. The results of plaque incision and venous grafting (Lue procedure) to correct the penile deformity of Peyronie's disease. BJU Int 2005; 95: 1029-33.

21 Kalsi J, Christopher AN, Ralph DJ, Minhas S. Plaque incision and fascia lata grafting in the surgical management of Peyronie's disease. BJU Int 2006; 98: 110-4.

22 Knoll LD. Use of small intestinal submucosa graft for the surgical management of Peyronie's disease. J Urol 2007; 178: 2474-8.

23 Breyer BN, Brant WO, Garcia MM, Bella AJ, Lue TF. Complications of porcine smal intestine submucosa graft for Peyronie's disease. J Urol 2007; 177: 589-91.

24 Hsu GL, Chen HS, Hsieh CH, Chen RM, Wen HS et al. Long-term results of autologous venous graft for penile morphological reconstruction. J Androl 2007; 28: 186-93.

25 Taylor FL, Levine LA. Surgical correction of Peyronie's disease using tunica albuginea plication or partial plaque excision with pericardial graft: long-term follow up. J Sex Med 2008; 5: 2221-8.

26 Cheung E, Clindinning E, Lessard L, Brock G. Five-year follow up of Peyronie's graft surgery: outcomes and patient satisfaction. J Sex Med 2011; 8: 594-600.

27 Gelbard MK. Relaxing incisions in the correction of penile deformity due to Peyronie's disease. J Urol 1995; 154: 1457-60. 